phys. stat. sol. (a) 164, 587 (1997)

Subject classification: 73.20.Dx; 73.40.Kp; S7.12

\title{
Magneto-Optical Study of Correlated Electron-Hole Layers in Single-Barrier Heterostructures
}

\author{
A. Parlangeli ${ }^{1}$ ) (a), P.C.M. Christianen (a), A.K. Geim (a), \\ J.C. MaAn (a), L. Eaves (b), and M. Henini (b) \\ (a) Research Institute for Materials, High Field Magnet Laboratory, \\ University of Nijmegen, Toernooiveld 1, NL-6525 ED Nijmegen, The Netherlands
}

(b) Department of Physics, University of Nottingham, Nottingham NG7 2RD, U.K.

(Received September 1, 1997)

We report the low-temperature photoluminescence investigation of a single-barrier GaAs/AlAs/ GaAs p-i-n heterostructure with applied magnetic fields of up to $17 \mathrm{~T}$. Under conditions of forward bias, electrons and holes accumulate at opposite sides of the barrier to form two coupled 2D layers of tunable density. We observe an onset of polarized recombination peaks originating from these layers when the filling factor is immediately lower than two. From the properties of the onset and the shift of the peaks with applied voltage we conclude that we have to take into account the Coulomb interaction between the layers to describe properly the physical situation of the system and we propose the picture of a correlated ground state to explain the experimental findings.

In the last decade a considerable interest has been addressed to the study of the optical properties of a two-dimensional electron gas (2DEG) in the regime of the Quantum Hall Effect (QHE) [1 to 5]. Among other properties, a strong depression of the photoluminescence (PL) from the lowest subband, an anomaly in peak position and the enhancement of polarization splitting were found at integer filling factor and explained in terms of suppression of screening of the photoexcited holes by the 2D electrons, occurring when the Fermi energy (FE) lies in the gap of the density of states (DOS) [1, 2].

In the present work we extend the experimental investigation to a system of correlated 2D electron-hole layers of equal densities, whose relative distance is comparable to the average intra-layer inter-particle distance, the GaAs Bohr radius [6] and the magnetic length (regime of the integer QHE). The ground state of such a system depends crucially on the strong inter-layer Coulomb coupling and can be expected to range from the indirect (superfluid) exciton state to the double Wigner crystal [7 to 9]. Indeed, we will show that a correlated ground state characterized by a binding energy of few meV stronger than the one of bulk GaAs exciton provides the most natural explanation of our experimental finding of an onset of PL peaks from the $2 \mathrm{D}$ layers for $v \lesssim 2$ and of their energy shift with density.

We realize the above-mentioned physical conditions in a $12 \mathrm{~nm}$ thick single barrier GaAs/AlAs/GaAs heterostructure under forward bias. In this situation, electrons and holes accumulate at opposite sides of the barrier to form two 2D gases of tunable den-

1) Tel.: 024-3 6530 52, Fax: 024-3 6524 40, e-mail: andrea@sci.kun.nl 
sity whose relative distance can be estimated by the Fang-Howard approach to be around $25 \mathrm{~nm}$, comparable with a GaAs Bohr radius of $15 \mathrm{~nm}$, an average interlayer distance of 15 to $30 \mathrm{~nm}$ (typical of our experimental conditions), and a magnetic length down to $7 \mathrm{~nm}$ at the highest fields we can reach $(17 \mathrm{~T})$. A very important advantage of our structure is that we can tune the density of the system by changing the applied voltage. In this way we can discriminate unambiguously the spectral features related to the 2D layers (bias dependent) from those of the bulk (bias independent). The layer composition of the $\mathrm{p}-\mathrm{i}-\mathrm{n}$ heterostructure discussed here is: $2 \mu \mathrm{m}$-doped GaAs $\left(4 \times 10^{18} \mathrm{~m}^{-2}\right), 100 \mathrm{~nm}$ of weaker doping $\left(1 \times 10^{17} \mathrm{~m}^{-2}\right), 100 \mathrm{~nm}$ undoped GaAs, $11.9 \mathrm{~nm}$ AlAs barrier, $100 \mathrm{~nm}$ undoped GaAs, $100 \mathrm{~nm}$ n-doped GaAs $\left(2 \times 10^{16} \mathrm{~m}^{-2}\right)$, $500 \mathrm{~nm}$ n-doped GaAs $\left(4 \times 10^{18} \mathrm{~m}^{-2}\right)$. The sample was grown by molecular beam epitaxy on a [311] Si substrate and $\mathrm{Si}$ was used both as donor and as acceptor. All the main results shown here were carefully reproduced on an analogous sample grown on a [100] substrate where Be was used as acceptor, showing that the observed behavior is an intrinsic property of the coupled electron-hole system.

Measurements were performed at $4.2 \mathrm{~K}$ and in magnetic fields of up to $17 \mathrm{~T}$. Sweeping the magnetic field at fixed applied voltage, the electron tunneling current shows pronounced oscillations with a periodicity of $1 / B$, the minima occurring at integer filling factor [10]. From this analysis we determined the carrier density to range from 1 to $5 \times 10^{15} \mathrm{~m}^{-2}$ for applied voltages from 1.6 to $1.7 \mathrm{~V}$, or 150 to $250 \mathrm{mV}$ over flat band (FB).

Optical measurements were performed in the Faraday configuration and the PL was excited by a He-Ne laser and detected polarization resolved by a Jobin-Yvon monochromator and a GaAs photomultiplier. The PL spectra at zero magnetic field do not show any feature related to the $2 \mathrm{D}$ layers. In fact, increasing the applied voltage from reverse bias to $300 \mathrm{mV}$ forward bias we observe only the sharp onset at FB of the GaAs bulk exciton peak, whose energy position does not show any bias dependence. In a magnetic field we observe the growing of a shoulder at the low energy side of this peak in correspondence to $v=2$. Increasing further the magnetic field, this shoulder develops into a well resolved density dependent PL peak (Fig. 1) generated by recombination of carriers in the lowest confined subband with photoexcited carriers in the neighboring GaAs regions.

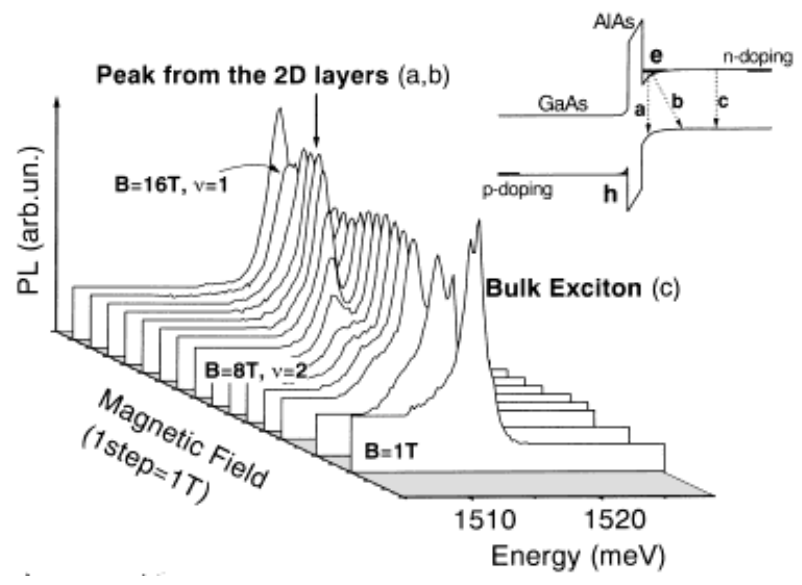

Fig. 1. Photoluminescence spectra in the $\sigma^{-}$polarization at $4.2 \mathrm{~K}$, $-1.65 \mathrm{~V}$ and magnetic fields from 1 to $17 \mathrm{~T}$. At $v=2(B=8 \mathrm{~T})$ a new peak arises few meV below the bulk exciton. In the insert the band diagram of the structure is shown, together with the possible recombination processes for electrons: 2D carriers with photoexcited ones in the bulk (direct recombination (a) and indirect recombination (b)), and the bulk exciton recombination (c). The same processes occur for holes 


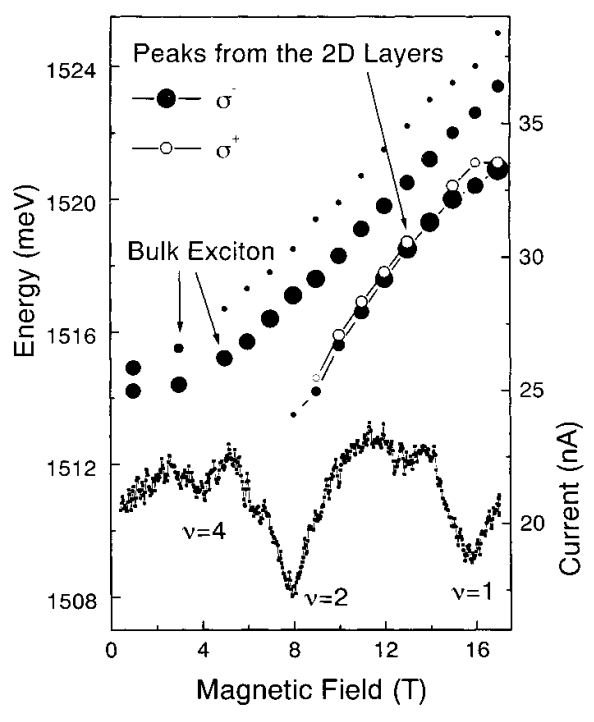

Fig. 2

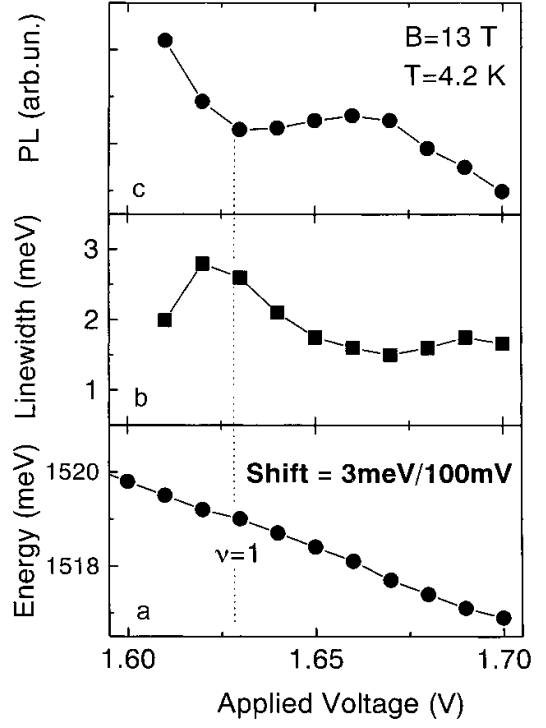

Fig. 3

Fig. 2. Upper part: Peak position (left ordinate) for both $\sigma^{-}$(filled circles) and $\sigma^{+}$(open circles) polarization as function of magnetic field. The bulk peaks are reported for comparison only in the $\sigma^{-}$configuration. The size of the circles is proportional to the peak intensity. Lower part: Magnetooscillations in the current (right ordinate) measured under the same conditions

Fig. 3. a) Peak position, b) linewidth and c) peak intensity at $B=13 \mathrm{~T}$ and $T=4.2 \mathrm{~K}$. Only the lineshape dependence on filling factor (b and c) can be directly understood as a property of the single layers, while inter-layer correlations can explain the shift with bias (a)

To the best of our knowledge such an onset was never reported up to now in measurements on single layers. We emphasize that $v=2$ is a very special situation for our coupled system because electrons and holes are confined by the magnetic field into orbits with a diameter equal to the intra-layer inter-particle distance, leading to a strong reduction of screening that occurs simultaneously for both types of carriers.

The fan plot of the peaks is reported in the upper part of Fig. 2 for both $\sigma^{-}$and $\sigma^{+}$ polarization configurations, together with the magneto-oscillations in the current at the same bias (Fig. 2, lower part) to identify the filling factor. The dependence on filling factor of lineshape, intensity and polarization splitting (Fig. 1 to 3) can be explained as a property of the single $2 \mathrm{D}$ layers [1 to 5]. We observe, anyway, that also the bulk exciton peak intensity depends on the filling factor of the quantized layers, and is maximum in correspondence to $v=2$ and $v=1$ (Fig. 1). A similar behavior was previously observed on single layer systems and attributed to population of the second confined subband $[1,4]$.

For any value of the magnetic field, the bulk peak does not depend on bias, while the other peak experiences a shift to lower energies $\Delta \mathrm{E} / \Delta \varrho$ of about $0.7 \mathrm{meV} / 10^{15} \mathrm{~m}^{-2}$ ( $3 \mathrm{meV} / 100 \mathrm{mV}$ applied), as shown in Fig. 3 for $B=13 \mathrm{~T}$. We cannot compare this quantity with any measurement on single layers, since a systematic investigation of these systems as a function of density has not been reported so far, but we argue that it is necessary to take into account the coupling between the two layers in order to explain 
this behavior. In fact, we notice that at $v \leq 2$ the FE lies on the lowest LL and therefore recombination from the 2D layers can occur only from the FE, at least within the linewidth of the LL that is not expected to exceed $1 \mathrm{meV}$. The possible recombination processes with the photoexcited carriers in the bulk are shown in the insert of Fig. 1. The spatially direct recombination (a) occurs at an energy that increases when the applied voltage is increased, because of the stronger band bending, while recombination with photoexcited carriers in the bulk regions without band bending (process b) would be bias independent like the purely bulk one (c).

To understand the experimental results, we propose a picture based on a correlated inter-layer ground state arising at $v \leq 2$ in correspondence with the suppression of screening by magnetic quantization. In this scheme, the carriers in the lowest LL (pinned to the FE) recombine radiatively with the photoexcited ones in the GaAs bulk regions at expense of the binding energy of the correlated state. The binding energy would be slightly stronger than the GaAs bulk exciton one and would depend on both density and filling factor; according to our data, it would increase with density at fixed magnetic field with a rate of $0.7 \mathrm{meV} / 10^{15} \mathrm{~m}^{-2}$. This fascinating interpretation is able to explain most of the observed features in a more natural way than the single particle picture can do, but it certainly needs further experimental support and a deeper theoretical understanding.

In conclusion, we have investigated the optical properties of coupled 2D layers of electrons and holes in the integer quantum Hall regime. This system experiences a strong suppression of screening at $v \lesssim 2$ and in correspondence to this value we have observed an onset of PL peaks originating from the recombination of the 2 D layers with photoexcited carriers in the bulk. We have shown the difficulties of the single particle picture to explain the shift of these peaks with bias and have proposed the picture of a correlated ground state of the system to explain the experimental results.

\section{References}

[1] A.J. Turberfield, S.R. Haynes, P.A. Right, R.A. Ford, R.G. Clark, J.F. Ryan, J.J. HARris, and C.T. Foxon, Phys. Rev. Lett. 65, 637 (1990).

[2] H. Buhmann, W. Joss, K. v. Klitzing, I.V. Kukushkin, G. Martinez, A.S. Plaut, K. Ploog, and V. B. Timofeev, Phys. Rev. Lett. 65, 1056 (1990).

[3] B.B. Goldberg, D. Heimann, A. Pinczuk, L. Pfeiffer, and K. West, Phys. Rev. Lett. 65, 641 (1990).

[4] I.V. Kukushkin, N.J. Pulsford, K. v. Klitzing, K. Ploog, and V.B. Timofeev, Surf. Sci. 263, 30 (1992)

[5] A review can be found in I.V. Kukushin, and V.B. Timofeev, Adv. Phys. 45, 147 (1996).

[6] B.R.A. Neves et al., Proc. 23rd Internat. Conf. Phys. Semiconductors, Berlin 1996, Ed. M. Scheffler and R. Zimmermann, World Scientific Publ. Co., Singapore 1996 (p. 2275).

[7] J. Szymanski, L. Swierkowski, and D. Neilson, Phys. Rev. B 50, 11002 (1994).

[8] D. Yoshioka and A.H. MacDonald, J. Phys. Soc. Japan 59, 4211 (1990).

[9] X.M. Chen and J.J. Quinn, Phys. Rev. Lett. 67, 895 (1991).

[10] E. Böckenhoff, K. v. Klitzing, and K. Ploog, Phys. Rev. B 38, 10120 (1988). 